\title{
EFECTO DE DIFERENTES TIPOS DE ENTRENAMIENTO FÍSICO EN LA ALTURA DE SALTO DE GIMNASTAS: META-ANÁLISIS
}

\author{
EFFECT OF DIFFERENT TYPES OF PHYSICAL TRAINING ON THE HEIGHT OF \\ GYMNAST JUMP: META-ANALYSIS
}

\section{EFEITO DE DIFERENTES TIPOS DE TREINAMENTO FíSICO NA ALTURA DO SALTO DO GINÁSTICO: META-ANÁLISE}

\author{
Paul Ulloa-Sánchez ${ }^{1}$, Jessenia Hernández Elizondo ${ }^{1,2,3}$ \\ piuulloa@gmail.com; jessenia.hernandez@ucr.ac.cr \\ ${ }^{1}$ Escuela de Educación Física y Deportes, Universidad de Costa Rica, San José, Costa \\ Rica. \\ ${ }^{2}$ Posgrado en Ciencias del Movimiento Humano, Universidad de Costa Rica, San José, \\ Costa Rica. \\ ${ }^{3}$ Centro de Investigación en Ciencias del Movimiento Humano, Universidad de Costa \\ Rica, San José, Costa Rica.
}

Envío Original: 2020-07-23 Reenviado: 2021-03-02 Aceptado: 2021-03-16

Publicado: 2021-04-06

Doi: https://doi.org/10.15517/pensarmov.v19i1.43155

\begin{abstract}
RESUMEN
El principal objetivo del presente estudio fue determinar el efecto de diferentes tipos de entrenamiento físico en la altura de salto de gimnastas, bajo la metodología de metaanálisis. Se siguió los lineamientos PRISMA, las bases de datos electrónicas consultadas en la búsqueda fueron EBSCOhost (SPORTDiscus, MEDLINE, Academic Search Complete, E-Journals, OmniFile Full Text Select (H.W. Wilson), Web of Science, Scopus, SciencDirect, Research Gate y Pubmed para identificar los artículos relevantes que investigaron la aplicación de un entrenamiento físico y evaluaron la altura salto en gimnastas antes y después de la intervención. Después del proceso de selección de estudios y a partir de los criterios de inclusión, se codificaron 29 estudios individuales y se
\end{abstract}


codificaron 157 tamaños de efecto (TE) individuales. La muestra total de sujetos fue 688 gimnastas. El grupo experimental mostró un TE global de bajo a moderado y significativo $\left(T E=0.33, p<0.001^{*}\right)$. Se evidenció una diferencia significativa $\left(p=p<0.001^{*}\right)$ entre los tipos de entrenamiento; el análisis Post Hoc mostró que el TE global del entrenamiento de pliometría $\left(T E=0.85, p<0.001^{*}\right)$ fue mayor al de estiramiento $(T E=-0.07, p=0.56)$ y "otros" $\left(T E=0.48, p<0.001^{*}\right)$, pero no al de vibración $\left(T E=0.54, p<0.001^{*}\right)$; además, el TE global de tipo de estiramiento fue significativamente menor a todos los tipos de entrenamiento. Se concluyó que el tipo de entrenamiento que mostró el mayor TE global fue pliometría, seguido por el entrenamiento en plataforma vibratoria. Parece que el estiramiento no se relaciona significativamente con la altura de salto de gimnastas.

Palabras clave: gimnasia, altura de salto, entrenamiento físico y acondicionamiento.

\begin{abstract}
The main objective of the present study was to determine the effect of different types of physical training on the jumping height of gymnasts, under the meta-analysis methodology. The PRISMA guidelines were followed, the electronic databases consulted in the search were EBSCOhost (SPORTDiscus, MEDLINE, Academic Search Complete, E-Journals, OmniFile Full Text Select (HW Wilson), Web of Science, Scopus, SciencDirect, Research Gate and Pubmed to identify relevant articles that investigated the application of physical training and evaluated jump height in gymnasts before and after the intervention. After the study selection process and based on the inclusion criteria, 29 individual studies were coded and coded 157 individual effect sizes (TE). The total sample of subjects was 688 gymnasts. The experimental group showed a global TE of low to moderate and significant (TE $=0.33$, $\left.p<0.001^{*}\right)$. A significant difference was evidenced $\left(p=p<0.001{ }^{*}\right)$ between the types of training; Post Hoc analysis showed that the global TE of plyometrics training (TE $=0.85, p$ $<0.001^{*}$ ) was higher than that of stretching. or (TE $\left.=-0.07, p=0.56\right)$ and "others" (TE = $0.48, p<0.001^{*}$ ), but not to vibration (TE $\left.=0.54, p<0.001^{*}\right)$; furthermore, the overall stretch type TE was significantly lower than all types of training. It was concluded that the type of training that showed the highest overall TE was plyometrics, followed by vibrational platform training. Stretching does not appear to be significantly related to gymnasts' jumping height.
\end{abstract}

Keywords: gymnastics, jump height, physical training \& conditioning. 


\section{RESUMO}

O objetivo principal do presente estudo foi determinar o efeito de diferentes tipos de treinamento físico na altura de salto de ginastas, por meio da metodologia de metanálise. As diretrizes do PRISMA foram seguidas, as bases de dados eletrônicas consultadas na busca foram EBSCOhost (SPORTDiscus, MEDLINE, Academic Search Complete, EJournals, OmniFile Full Text Select (HW Wilson), Web of Science, Scopus, SciencDirect, Research Gate e Pubmed to identificar artigos relevantes que investiguem a aplicação do treinamento físico e avaliem a altura do salto em ginastas antes e após a intervenção.Após o processo de seleção dos estudos e com base nos critérios de inclusão, 29 estudos individuais foram codificados e codificados com 157 tamanhos de efeito individual (TE). a amostra total de sujeitos foi de 688 ginastas. O grupo experimental apresentou TE global de baixo a moderado e significativo $\left(T E=0,33, p<0,001^{*}\right)$. Diferença significativa $(p=p$ $<0,001^{*}$ ) entre os tipos de treinamento; Pós A análise Hoc mostrou que o TE global do treinamento pliométrico $\left(T E=0,85, p<0,001^{*}\right.$ ) foi maior do que o do alongamento. ou (TE $=-0,07, p=0,56)$ e "outros" (TE = 0,48, $\left.p<0,001^{*}\right)$, mas não para vibração (TE = 0,54, $p$ $\left.<0,001^{*}\right)$; além disso, o tipo geral de alongamento TE foi significativamente menor do que todos os tipos de treinamento. Concluiu-se que o tipo de treinamento que apresentou maior TE geral foi a pliometria, seguida do treinamento em plataforma vibracional. $O$ alongamento não parece estar significativamente relacionado à altura de salto das ginastas.

Palavras-chave: ginástica, salto em altura, treinamento físico e condicionamento,

\section{INTRODUCCIÓN}

La gimnasia es un deporte olímpico que se divide en diferentes especialidades, como la gimnasia artística, la gimnasia rítmica y la gimnasia de trampolín. En general, los deportistas de esta disciplina poseen habilidades acrobáticas, con una técnica depurada alcanzando la perfección, pues es un deporte estético, es decir, cada detalle de sus gestos deportivos suma a la nota final de cada rutina tomando en cuenta detalles como el nivel de dificultad.

En las modalidades gimnásticas destaca la fuerza explosiva, que en el caso de la gimnasia rítmica se demuestra en elementos como las zancadas, y el caso de la gimnasia artística resulta muy importante en elementos como saltos y mortales, sobre todo en la barra de equilibrios (Leyton, Campo, Sabido, y Morenas, 2015). En este sentido, se comprende 
que la fuerza explosiva es una capacidad física determinante en esta disciplina deportiva, ya que es necesaria para una correcta ejecución de dichos movimientos gimnásticos.

La fuerza explosiva es la capacidad física de generar una mayor fuerza muscular en un menor tiempo sin pérdida de eficacia (Romero, Aymara, y Rojas, 2020). La valoración de la fuerza explosiva se puede determinar mediante pruebas dinámicas e isométricas. Entre las valoraciones dinámicas destacan los distintos test de salto. Así mismo, la fuerza explosiva se puede observar en la altura de salto que es la cantidad en centímetros que se despega del suelo una persona realizando un salto (Pérez y Llana, 2015).

Conocer el efecto de diferentes tipos de entrenamiento físico sobre la altura de salto de gimnastas, se justifica por el hecho de que el rendimiento se puede evaluar a partir de esta capacidad física, pues Bradshaw y Le Rossignol (2004) mencionan que existe una correlación directa, moderada, y significativa entre resultados tanto en el aparato "piso" y los saltos tipo squat jump (SJ: $r=0.76, p<0.05^{*}$ ) y countermovement jump (CMJ: $r=0.75$, $p<0.05^{*}$ ), como en "salto al potro" (SJ: $r=0.62, p<0.05^{*}$; CMJ: $r=0.63, p<0.05^{*}$ ), para el caso de la gimnasia artística.

En la actualidad ya existen publicaciones de diferentes autores que muestran el efecto que tienen algunos tipos de entrenamientos físicos sobre la altura de salto en gimnastas, por ejemplo: el entrenamiento pliométrico, diferentes tipos de estiramiento (estiramiento estático (EE), estiramiento dinámico (ED) y estiramiento de facilitación neuromuscular propioceptiva (FNP)) (Dallas, Smirniotou, et al., 2014; Harper, 2011; Silva et al., 2018), entrenamiento en plataforma vibratoria (EV), entrenamiento en trampolín (ET), entrenamiento contra resistencia y entrenamiento propioceptivo. Adicionalmente, existen dos estudios que evidenciaron los efectos de un tipo de intervención basado en entrenamiento pliométrico como método para potenciación post-activación con diseños de efecto agudo (Dallas, Pappas, Ntallas, y Paradisis, 2018; Dallas, Mavvidis, Kosmadaki, Tsoumani y Dallas, 2019).

Conceptualmente y a manera de resumen, se pueden definir las diferentes intervenciones de ejercicio encontradas en la literatura de la siguiente manera:

(1) Entrenamiento pliométrico: se basa en la ejecución de ejercicios que consisten en la aceleración y desaceleración rápida de los músculos, creando un ciclo de acortamiento-estiramiento (Chu y Myer, 2016).

(2) Estiramientos: consiste en elongar un músculo o un grupo muscular mediante un movimiento articular específico, sus categorías se diferencian principalmente por el tiempo de elongación, se clasifican en: movimientos balísticos, pasivos (con ayuda 
de otra persona) y activos (sin ayuda de otra persona) (Montalvo y Dorgo, 2019; Papia, Bogdanis, Toubekis, Donti y Donti, 2018; Silva et al., 2018).

(3) Entrenamiento en plataforma vibratoria: se caracteriza por la ejecución de diferentes tipos de saltos o el movimiento de sentadilla (squat) en fases excéntricas y concéntricas sobre una plataforma vibratoria (Dallas, Mavvidis et al., 2019; Tsopani et al., 2014).

(4) Entrenamiento en trampolín: consiste en la ejecución de diferentes tipos de saltos a máxima potencia de miembros inferiores, sobre una superficie fundamentalmente constituida por una lona elástica y resortes que facilitan elevar considerablemente el centro de masa del ejecutante (Ulloa y Hernández, 2020).

(5) Entrenamiento con contra resistencia: este se basa en la ejecución de ejercicios en contra de una resistencia externa (pesas, peso corporal, liga, entre otros) (Piazza et al., 2014).

(6) Entrenamiento propioceptivo: este se enfoca en la ejecución de ejercicios que estimulen la actividad de los órganos propioceptores, específicamente husos musculares, órganos del tendinosos de Golgi y corpúsculos de Pacini, utilizando materiales como balance board, pelotas de pilates, entre otros (Dobrijević, Moskovljević, Marković y Dabović, 2018).

A pesar de que ya existen estudios que han examinado el efecto de estos tipos de entrenamientos físicos en la altura de salto de gimnastas, hasta la actualidad no se conoce ningún estudio científico de tipo revisión sistemática o meta-análisis que resuma esta información, por lo que el objetivo del presente estudio es determinar el efecto de diferentes tipos de entrenamiento físico en altura de salto de gimnastas, bajo la metodología de metaanálisis.

\section{METODOLOGÍA}

El presente meta-análisis se realizó siguiendo los lineamientos generales para el reporte de revisiones sistemáticas y meta-análisis PRISMA (por sus siglas en inglés) (Liberati et al., 2009).

\section{Estrategia de búsqueda}

Los estudios fueron localizados por medio de búsqueda en base de datos electrónicas, búsqueda en listas de referencias de artículos y en revistas especializadas en el tema, publicados hasta mayo de 2020. Las bases de datos utilizadas en la búsqueda 
fueron: EBSCOhost (SPORTDiscus), MEDLINE, Academic Search Complete, E-Journals, OmniFile Full Text Select (H.W. Wilson), Web of Science, Scopus, SciencDirect, Research Gate y Pubmed para identificar los artículos relevantes que investigaron la aplicación de un entrenamiento físico y evaluaron la altura salto antes y después del mismo, y en donde los sujetos fueron gimnastas. Se utilizó la siguiente frase boleana en inglés y en español: ("vertical jump" OR "jumping" OR "explosive strength of lower extremities" OR "jump performance" OR "jump height" OR "high jump") AND (plyometrics OR training OR treatment OR "jumping techniques" OR “exercise") AND (gymnastics OR gymnasts).

\section{Criterios de legibilidad}

Los estudios debían tener un diseño experimental, cuasiexperimental o preexperimental, en idioma inglés o español; el tratamiento tuvo que ser aplicado en gimnastas de ambos sexos; por último, en los resultados debió reportarse el promedio y desviación estándar de la altura de salto por grupo en el pre-test y post-test. Los estudios con una intervención de tipo psicológica no se incluyeron.

En la figura 1 se muestra el flujograma del proceso de búsqueda, selección, eliminación e inclusión de los estudios que cumplieron con los criterios de elegibilidad. Los 29 estudios codificados suman una muestra total de 688 sujetos, de los cuales 614 (89.2\%) son gimnastas de alto rendimiento o de élite y 74 (10.8\%) son gimnastas de nivel básico. 
Se eliminaron 494 estudios

- No presentaron un entrenamiento físico para altura de salto

- No fueron aplicados en gimnastas

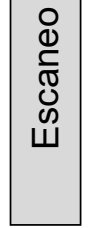

Se revisaron 67 artículos completos

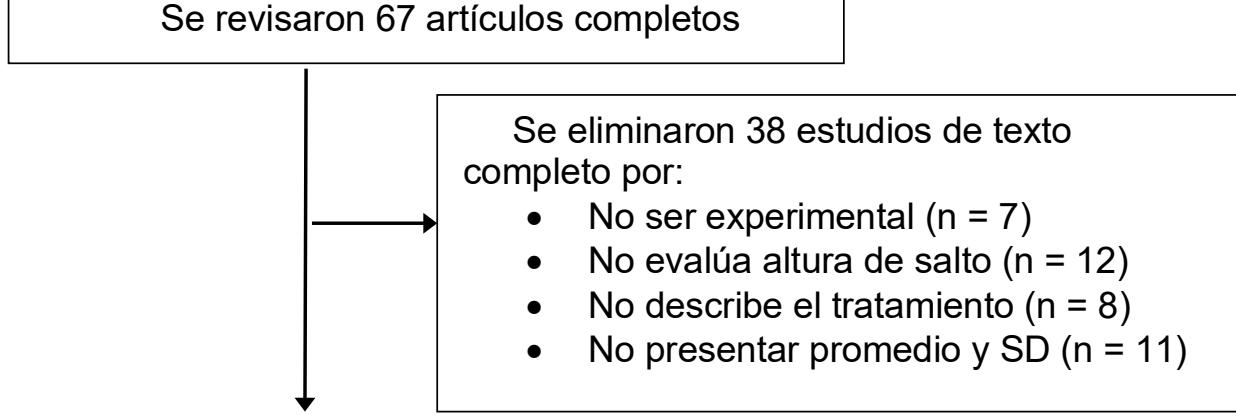

Estudios incluidos para el meta - análisis $(n=29)$

Figura 1. Flujograma para la selección de estudios incluidos. Fuente: elaboración propia basado en los lineamientos PRISMA.

\section{Evaluación de la calidad metodológica de los estudios}

Se confeccionó una escala para evaluar la calidad del diseño de un estudio individual, según las características de un diseño que presenta validez interna (Campbell y Stanley, 1970). La escala evaluó 4 criterios: (1) presenta medición pretest y post-test, (2) presenta aleatorización de los grupos, (3) presenta grupo control y (4) reporta muerte experimental. Si el criterio está presente se otorga un punto y si no está presente se otorga un cero. El puntaje máximo a obtener es de cuatro puntos. 


\section{Análisis estadístico}

El cálculo de cada tamaño de efecto (TE) se realizó a partir de la diferencia entre medias estandarizadas, utilizando la fórmula de delta (ver figura 2, fórmula 1) propuesta por Glass (Thomas y French, 1986) y su varianza (ver figura 2, fórmula 2). Este TE indica el cambio en el desempeño de cada grupo a lo largo de las mediciones. En donde, $\mathrm{M}_{1}$ es el promedio del postest, $\mathrm{M}_{2}$ es el promedio del pretest, $\mathrm{DE}_{2}$ es la desviación estándar del pretest $y, n_{1}$ y $n_{2}$ se sustituyen por el tamaño de la muestra $(n)$ de cada grupo.

$$
T E=\frac{M_{1}-M_{2}^{(1)}}{D E_{2}} \quad \operatorname{var} T E=\frac{n_{1}+n_{2}}{n_{1} n_{2}}+\frac{T E^{2}}{2\left(n_{1}+n_{2}\right)}
$$

Figura 2. Fórmulas tamaño de efecto individual para el diseño intragrupo. Fuente: elaboración propia.

También se calculó el tamaño del efecto de trabajos archivados, se realizó análisis de sesgo general por medio de la regresión de Egger y análisis de heterogeneidad; adicionalmente, se realizó el análisis de variables moderadoras.

Para cada nivel de las variables moderadoras se obtuvo un intervalo de confianza y se estableció la significancia del TE. Una vez realizada las pruebas de normalidad Kolmogorov-Smirnov (K-S), para determinar el efecto de las variables moderadoras continuas se utilizó la técnica de correlación de Pearson, para variables que contienen más de 30 TE, para las variables con menos de 30 TE se utilizó la técnica de correlación de Spearman. Adicionalmente, también se utilizó la técnica de Pearson para determinar la relación entre la calidad de los estudios y los TE. Por último, para las variables categóricas se utilizó análisis de varianza (ANOVA) de una vía, para las variables que mostraron diferencias significativas, se les realizó el análisis Post Hoc de Tuckey. Para la codificación y análisis estadísticos se utilizaron los programas Microsoft Excel 2019 y el paquete estadístico IBM-SPSS Statistics 25.

\section{Variables moderadoras}

Debido a que el propósito de este estudio es determinar el efecto de diferentes tipos de entrenamientos físicos, los TE de cada tipo de entrenamiento se agruparon y se analizaron como variables moderadoras categóricas.

Los tipos de entrenamiento que se agruparon son: pliometría, estiramiento, vibración y "otros". En "otros" se agruparon las intervenciones que utilizaron como tratamiento el 
entrenamiento en trampolín (ET), entrenamiento con cinturones de peso (weighted belts), entrenamiento contra resistencia, entrenamiento propioceptivo y potenciación postactivación, debido a que estos tipos de entrenamiento no presentaron la suficiente cantidad de TE para analizarlas por separado.

\section{Organización de la información}

Las características principales de los estudios individuales incluidos en el presente meta-análisis se detallan en los anexos $\underline{1}, \underline{2}, \underline{3}, \underline{4}, \underline{5}$ y $\underline{6}$, dicha información se agrupó por tipo de entrenamiento, los cuales poseen variables particulares, por lo que los indicadores de las tablas se ajustaron a ello.

\section{RESULTADOS}

En la tabla 1 se muestra la estadística descriptiva del grupo experimental y grupo control, en la misma se evidencia que el grupo experimental mostró un TE global de bajo a moderado, significativo $\left(p<0.001^{*}\right)$ y con una alta heterogeneidad; por otro lado, el grupo control mostró un TE global bajo, no significativo $(p=0.62)$ y con baja heterogeneidad.

Tabla 1.

Estadística descriptiva según el grupo experimental y grupo de control

\begin{tabular}{lcccccc}
\hline \multicolumn{1}{c}{ Grupo } & $\mathbf{N}$ & TE & IC (95\%) & $\mathbf{p}$ & $\mathbf{Q}(\mathbf{p})$ & $\mathbf{I}^{\mathbf{2}} \mathbf{( \% )}$ \\
\hline Experimental & 118 & 0.33 & $0.22-0.44$ & $<0.001^{*}$ & $361.02\left(<0.001^{*}\right)$ & 67.6 \\
Control & 39 & 0.18 & $0.07-0.29$ & 0.62 & $34.87(0.62)$ & 0
\end{tabular}

Nota: $\mathrm{N}$ = número de tamaños de efecto; TE = tamaños de efecto global, IC = intervalo de confianza, $\mathrm{p}=$ significancia, $\mathrm{Q}=$ prueba de heterogeneidad; $\mathrm{I}^{2}=$ prueba de inconsistencia. Fuente: elaboración propia.

En la figura 3 se muestra mediante el gráfico de forest plot el comportamiento de la dispersión de los TE del grupo experimental. De acuerdo a los resultados del análisis de sesgo general por medio del gráfico de embudo (funnel plot, figura 3 ) complementado con la regresión de Egger $\left(t=5.13\right.$ y $\left.p<0.001^{*}\right)$, se concluye que hay sesgo general en el metaanálisis. El análisis de sesgo de publicación mediante la fórmula de trabajos archivados (K0) evidenció que son necesarios 76.8 TE no significativos para que el TE global del grupo experimental resulte no significativo. 


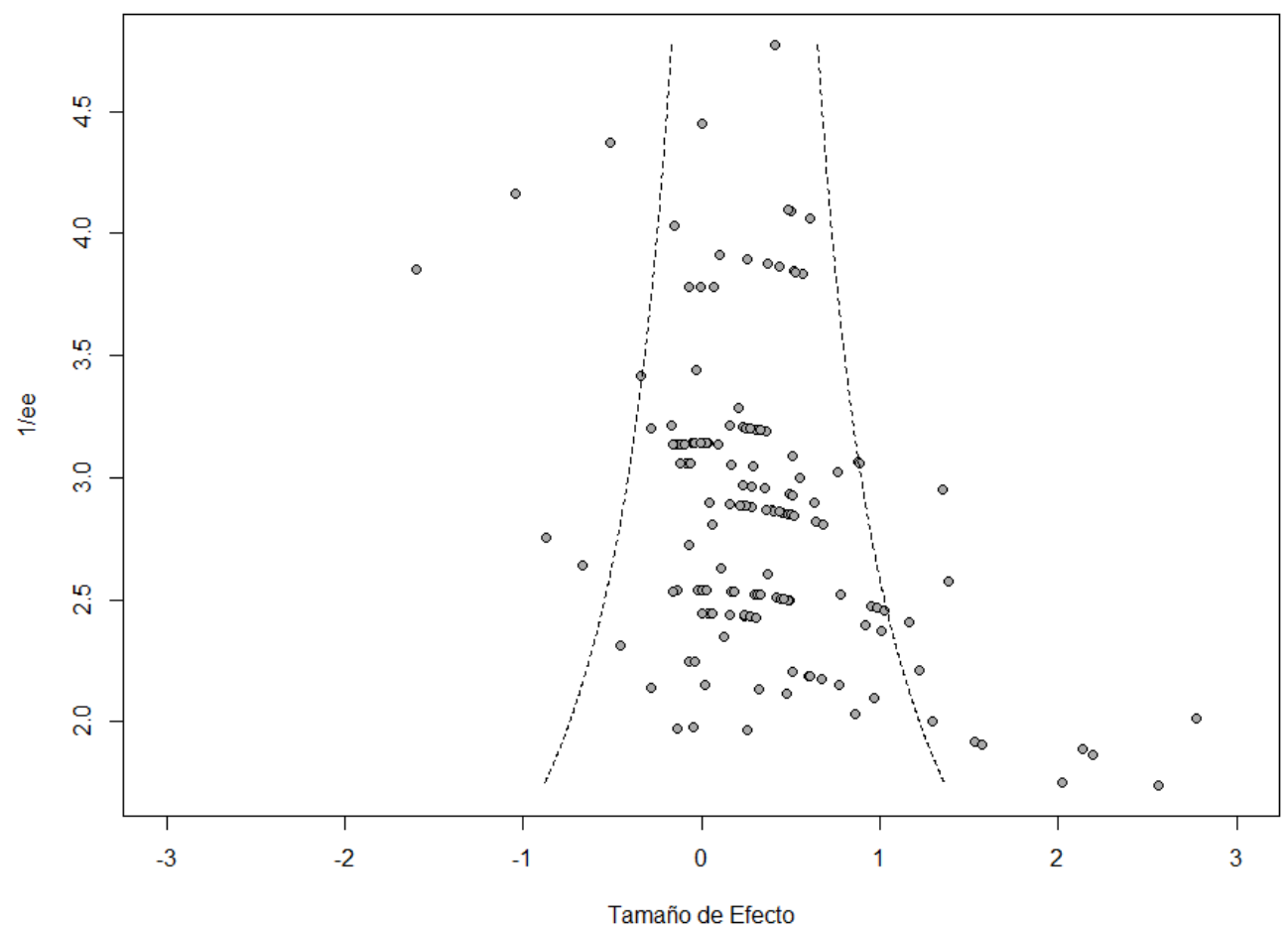

Figura 3. Sesgo general (funnel plot). Fuente: elaboración propia basado en PRISMA.

En cuanto a las variables moderadoras generales (tabla 2), se muestra que no se encontró correlación significativa $(p>0.05)$ entre la edad, masa corporal y talla y los TE. Así mismo, no se evidenció una correlación entre los TE y la calidad metodológica de los estudios ( $r=0.04 ; p=0.7$ ), esto sugiere que la calidad de los estudios no afecta los resultados del presente meta-análisis. Por otro lado, el análisis de varianza no mostró diferencias significativas $(p>0.05)$ entre sexo, pero sí mostró diferencias significativas $\left(p<0.001^{*}\right)$ entre diseños, y tipo de entrenamiento. 
Tabla 2.

Datos descriptivos y análisis de variables moderadoras generales

\begin{tabular}{|c|c|c|c|c|c|c|c|c|}
\hline \multicolumn{2}{|c|}{ Variable moderadora } & \multirow{2}{*}{$\begin{array}{c}\mathbf{N} \\
118\end{array}$} & \multirow{2}{*}{$\begin{array}{c}\text { TE } \\
0.33\end{array}$} & \multirow{2}{*}{$\begin{array}{c}\text { IC (95\%) } \\
0.22-0.44\end{array}$} & \multirow{2}{*}{$\begin{array}{c}\mathbf{p} \\
<0.001^{*}\end{array}$} & \multirow[t]{2}{*}{$\mathbf{F}$} & \multirow{2}{*}{$\begin{array}{c}\mathbf{r} \\
-0.03\end{array}$} & \multirow{2}{*}{$\begin{array}{c}\mathbf{p} \\
0.73\end{array}$} \\
\hline Edad (años) & Rango: $8.1-24.4$ & & & & & & & \\
\hline $\begin{array}{l}\text { Masa corporal } \\
(\mathrm{kg})\end{array}$ & $\begin{array}{l}\text { Rango: } 28.7- \\
73.05\end{array}$ & 103 & 0.27 & $0.15-0.38$ & $<0.001^{*}$ & & 0.17 & 0.86 \\
\hline Talla (cm) & $\begin{array}{l}\text { Rango: } 129.3- \\
176\end{array}$ & 107 & 0.28 & $0.17-0.39$ & $<0.001^{*}$ & & 0.18 & 0.07 \\
\hline $\begin{array}{l}\text { Calidad de los } \\
\text { estudios }\end{array}$ & & 118 & 0.33 & $0.22-0.44$ & $<0.001^{*}$ & & 0.04 & 0.70 \\
\hline \multirow[t]{4}{*}{ Sexo } & & 118 & 0.33 & $0.22-0.44$ & $<0.001^{*}$ & 1.91 & & 0.15 \\
\hline & Femenino & 77 & 0.29 & $0.12-0.45$ & $0.002^{*}$ & & & \\
\hline & Masculino & 13 & 0.41 & $0.14-0.67$ & $0.008^{*}$ & & & \\
\hline & Mixto & 28 & 0.02 & $\begin{array}{c}-0.06- \\
0.11\end{array}$ & 0.7 & & & \\
\hline \multirow[t]{4}{*}{ Diseño } & & 118 & 0.33 & $0.22-0.44$ & $<0.001^{*}$ & 8.36 & & $<0.001^{*}$ \\
\hline & Experimental & 29 & 0.73 & $0.51-0.95$ & $<0.001^{*}$ & & & \\
\hline & Cuasiexperimental & 21 & 0.26 & $0.07-0.46$ & $0.02^{*}$ & & & \\
\hline & Pre-experimental & 68 & 0.2 & $0.06-0.34$ & $0.01^{*}$ & & & \\
\hline \multirow[t]{5}{*}{$\begin{array}{l}\text { Tipo de } \\
\text { entrenamiento }\end{array}$} & & 118 & 0.33 & $0.22-0.44$ & & 19.18 & & $<0.001^{*}$ \\
\hline & Pliometría & 19 & 0.85 & $0.60-1.10$ & $<0.001^{*}$ & & & \\
\hline & Estiramiento & 47 & -0.07 & $\begin{array}{c}-0.24- \\
0.09\end{array}$ & 0.56 & & & \\
\hline & Vibración & 19 & 0.54 & $0.37-0.72$ & $<0.001^{*}$ & & & \\
\hline & Otros & 32 & 0.48 & $0.36-0.60$ & $<0.001^{*}$ & & & \\
\hline
\end{tabular}

Nota: $\mathrm{N}$ = número de tamaños de efecto, TE = tamaño de efecto global; IC = intervalo de confianza; $p=$ significancia; $F=$ varianza de Fisher; $r=$ coeficiente de correlación. Fuente: elaboración propia. 


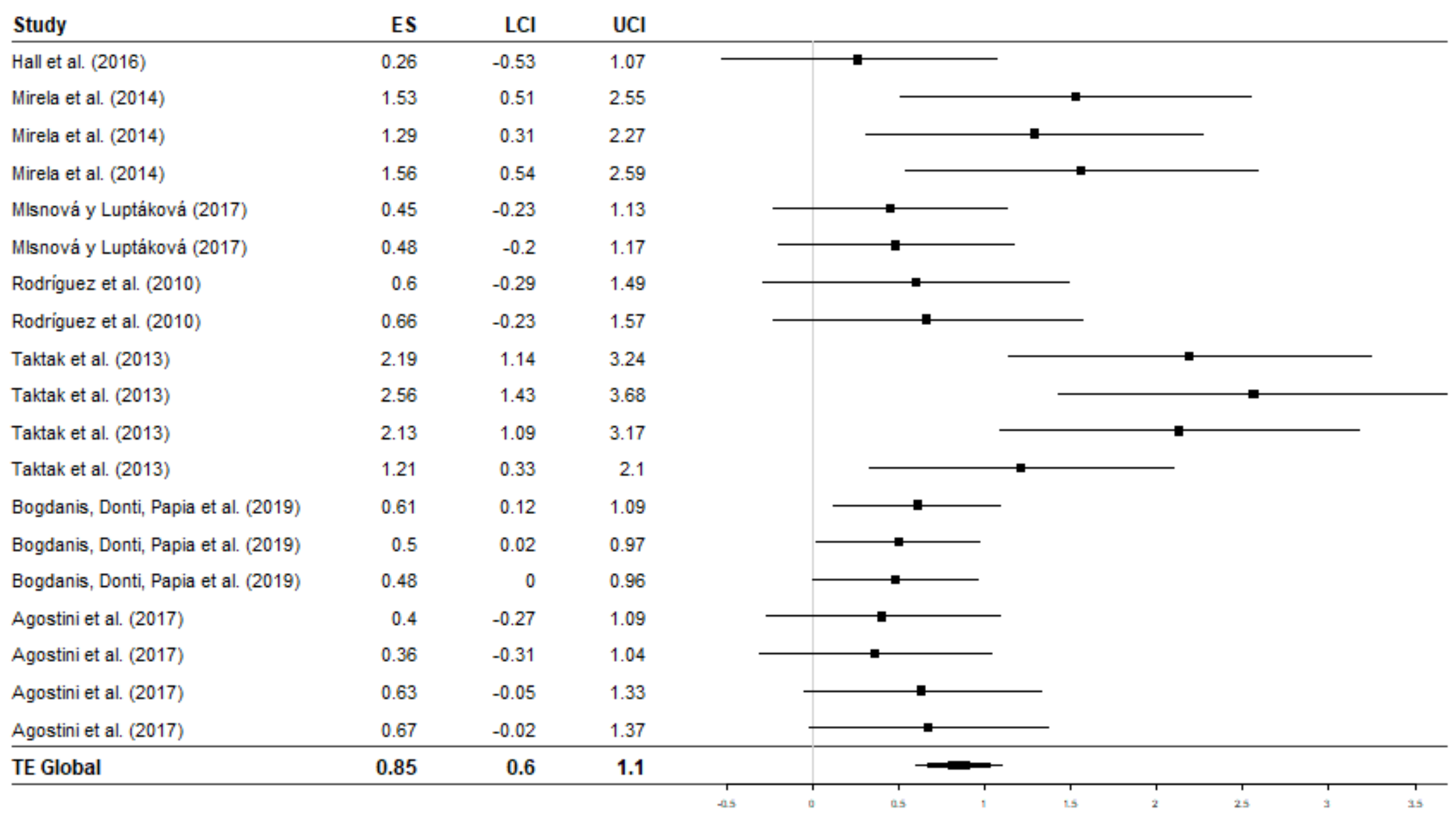

Figura 4. Forest plot del grupo experimental del tipo de entrenamiento pliometría (tamaño de efecto con su intervalo de confianza al 95\% entre la medición del pretest y post test). Fuente: elaboración propia basado en PRISMA. 
De acuerdo a los TE mostrados en la figura 4 y como se explica en la tabla 3 , para el tipo de entrenamiento pliometría, solo encontró una relación directa, de moderada a alta y significativa $\left(p<0.05^{*}\right)$ entre el rendimiento del salto vertical y el tiempo de descanso entre series y el volumen.

Tabla 3.

Análisis de variables moderadoras para el tipo de entrenamiento pliometría

\begin{tabular}{lccccccc}
\hline \multicolumn{1}{c}{ Variable moderadora } & $\mathbf{N}$ & Rango & TE & IC (95\%) & $\mathbf{p}$ & $\mathbf{r}$ & $\mathbf{p}$ \\
\hline Semanas & 19 & $4-38$ & 0.85 & $0.6-1.10$ & $0.003^{*}$ & -0.23 & 0.34 \\
Sesiones & 17 & $12-85$ & 0.88 & $0.61-1.16$ & $0.001^{*}$ & -0.18 & 0.49 \\
Series por sesión & 9 & $6-36$ & 0.6 & $0.42-0.76$ & 0.558 & 0.47 & 0.21 \\
Saltos por sesión & 9 & $93-185$ & 0.6 & $0.42-0.76$ & 0.558 & -0.1 & 0.8 \\
Minutos por sesión & 15 & $15-40$ & 0.79 & $0.65-1.27$ & $0.001^{*}$ & 0.32 & 0.25 \\
Descanso entre series (s) & 13 & $30-120$ & 0.83 & $0.68-1.40$ & $<0.001^{*}$ & 0.78 & $0.002^{*}$ \\
Volumen (min/intervención) & 15 & $220-1710$ & 0.79 & $0.65-1.27$ & $0.001^{*}$ & 0.6 & $0.018^{*}$ \\
\hline
\end{tabular}

Nota: $\mathrm{N}$ = número de tamaños de efecto, $\mathrm{TE}=$ tamaño de efecto global; $\mathrm{IC}=$ intervalo de confianza; $p$ = significancia; $r$ = coeficiente de correlación. Fuente: elaboración propia. 


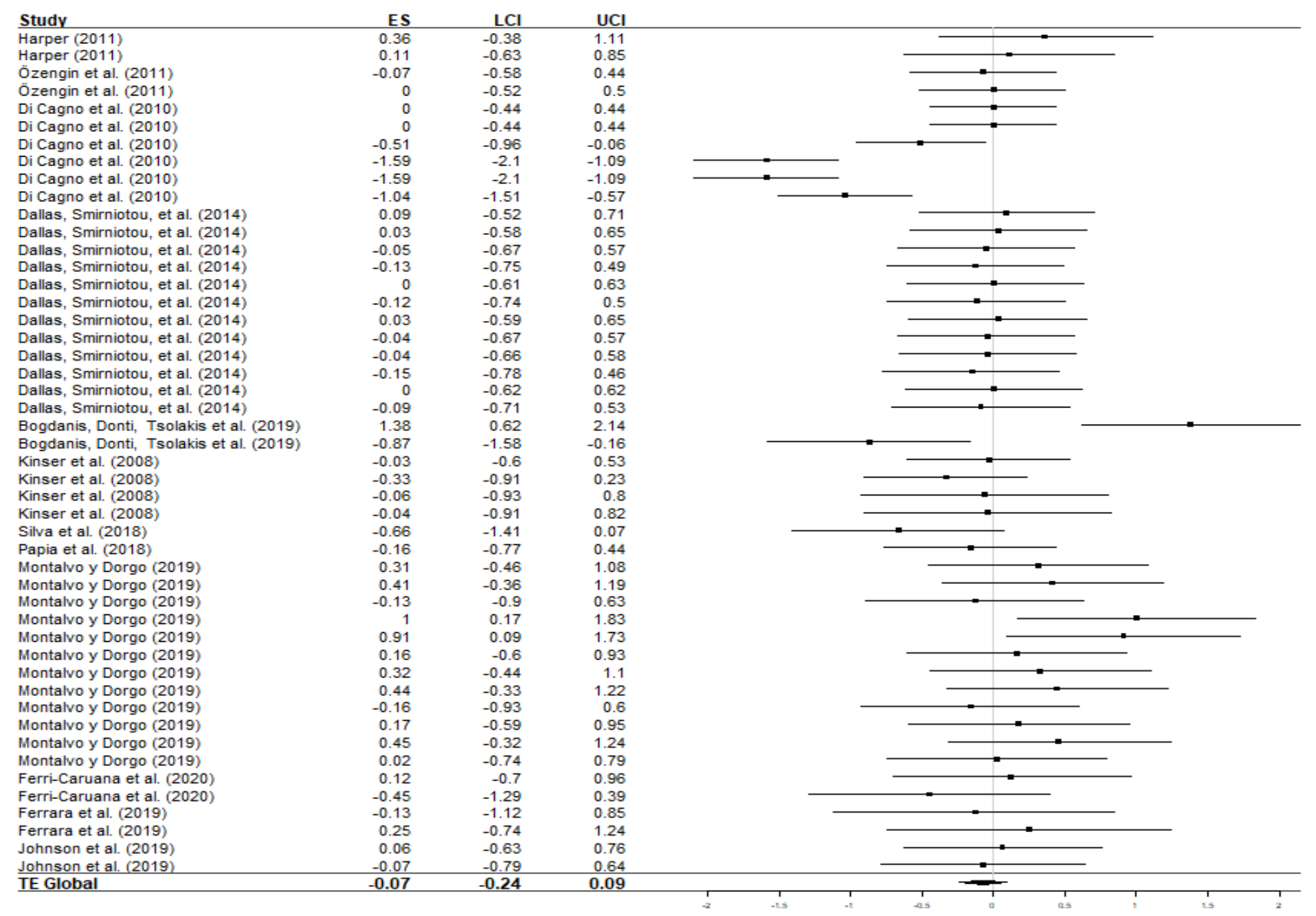

Figura 5. Forest plot del grupo experimental del tipo de entrenamiento estiramiento (tamaño de efecto con su intervalo de confianza al 95\% entre la medición del pretest y post test). Fuente: elaboración propia basado en PRISMA. 
Para el tipo de entrenamiento estiramiento (figura 5 y tabla 4), se encontró una relación inversa, moderada y significativa $\left(p=0.008^{*}\right)$ entre el rendimiento del salto vertical y las series por sesión; así mismo, se evidenció una relación directa, de baja a moderada y significativa $\left(p=0.018^{*}\right)$ entre el número de ejercicios.

Tabla 4.

Análisis de variables moderadoras para el tipo de entrenamiento estiramiento

\begin{tabular}{lccccccc}
\hline \multicolumn{1}{c}{ Variable moderadora } & $\mathbf{N}$ & Rango & TE & IC (95\%) & $\mathbf{p}$ & r & p \\
\hline Volumen & 34 & $15-700$ & -0.25 & $-0.38-0$ & 0.12 & -0.07 & 0.67 \\
(min/intervención) & 33 & $1-80$ & -0.25 & $-0.39--0.01$ & 0.11 & -0.45 & $0.008^{*}$ \\
Series por sesión & 34 & $10-90$ & -0.26 & $-0.39-0.01$ & 0.12 & -0.04 & 0.83 \\
Duración de la serie (s) & 29 & $5-120$ & -0.27 & $-0.42-0.02$ & 0.16 & -0.17 & 0.37 \\
Descanso entre series (s) & 44 & $1-30$ & -0.14 & $-0.23-0.11$ & 0.62 & 0.36 & $0.018^{*}$ \\
Número de ejercicios & 4
\end{tabular}

Nota: $\mathrm{N}$ = número de tamaños de efecto, TE = tamaño de efecto global; $\mathrm{IC}$ = intervalo de confianza; $p=$ significancia; $r=$ coeficiente de correlación. El rango del volumen (min/intervención) sin el estudio de Ferri-Caruana, Roig-Ballester, y Romagnoli (2020) es de 1.5-16 min, debido a que dicho estudio fue el único con un diseño de efecto crónico (4 semanas). Fuente: elaboración propia. 


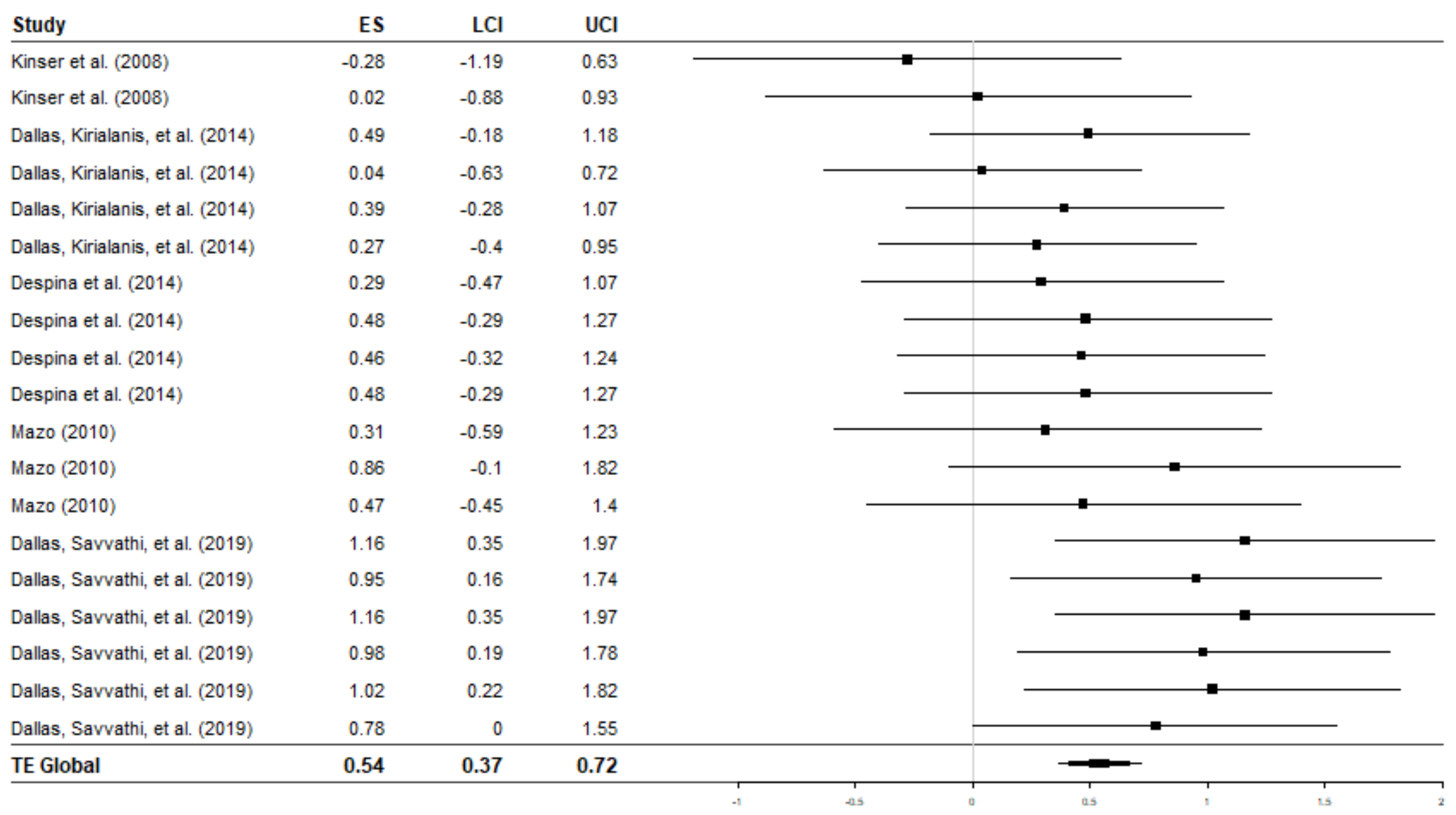

Figura 6. Forest plot del grupo experimental del tipo de entrenamiento vibración (tamaño de efecto con su intervalo de confianza al 95\% entre la medición del pretest y post test). Fuente: elaboración propia basado en PRISMA. 
En relación a los hallazgos del entrenamiento con vibración, los cuales se ilustran en la figura 6 y se explican estadísticamente en la tabla 5 , las sesiones y la media de desplazamiento tienen una relación directa, de moderada a alta y significativa $\left(p<0.05^{*}\right)$ con el rendimiento del salto vertical de gimnastas.

Tabla 5.

Análisis de variables moderadoras para el tipo de entrenamiento vibración

\begin{tabular}{lccccccc}
\hline \multicolumn{1}{c}{ Variable moderadora } & $\mathbf{N}$ & Rango & TE & IC (95\%) & $\mathbf{p}$ & $\mathbf{r}$ & $\mathbf{p}$ \\
\hline Sesiones & 19 & $1-18$ & 0.54 & $0.37-0.72$ & $<0.001^{*}$ & 0.8 & $<0.001^{*}$ \\
Minutos por sesión & 13 & $2-8$ & 0.33 & $0.2-0.44$ & 0.96 & -0.25 & 0.41 \\
Series por sesión & 19 & $1-32$ & 0.54 & $0.37-0.72$ & $<0.001^{*}$ & -0.3 & 0.21 \\
Duración de la serie (s) & 19 & $10-120$ & 0.54 & $0.37-0.72$ & $<0.001^{*}$ & -0.28 & 0.25 \\
Número de ejercicios & 19 & $1-5$ & 0.54 & $0.37-0.72$ & $<0.001^{*}$ & 0.13 & 0.59 \\
Hertz & 19 & $30-45$ & 0.54 & $0.37-0.72$ & $<0.001^{*}$ & -0.03 & 0.92 \\
Media de desplazamiento (mm) & 19 & $1.8-2.5$ & 0.54 & $0.37-0.72$ & $<0.001^{*}$ & 0.71 & $0.001^{*}$
\end{tabular}

Nota: $\mathrm{N}=$ número de tamaños de efecto, $\mathrm{TE}=$ tamaño de efecto global; $\mathrm{IC}=$ intervalo de confianza; $p$ = significancia; $r$ = coeficiente de correlación. Fuente: elaboración propia. 


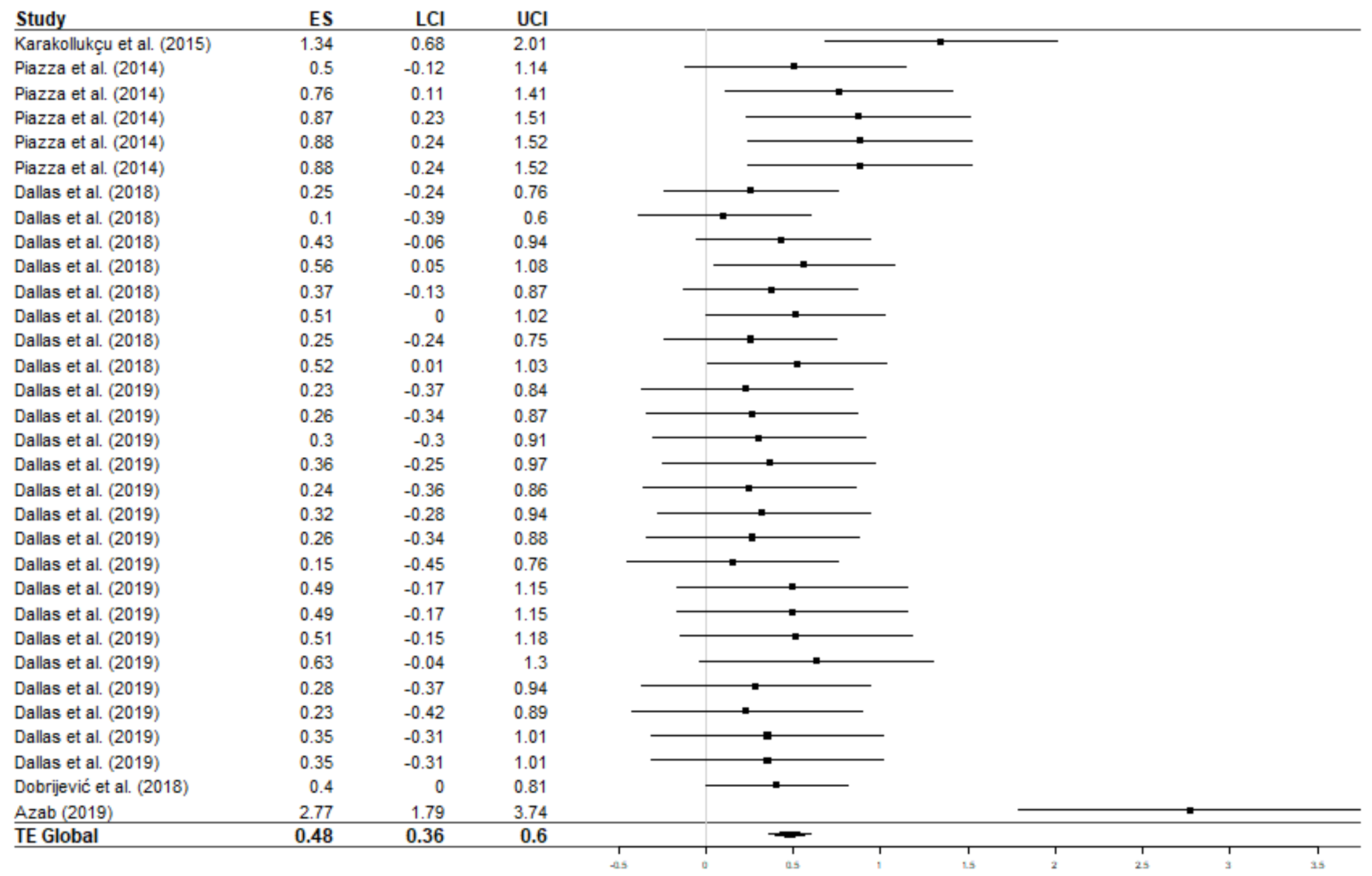

Figura 7. Forest plot del grupo experimental de la categoría "otros" (tamaño de efecto con su intervalo de confianza al 95\% entre la medición del pretest y post test). Fuente: elaboración propia basado en PRISMA. 


\section{DISCUSIÓN}

El propósito principal de este meta-análisis fue determinar el efecto de diferentes tipos de entrenamiento físico en altura de salto de gimnastas.

Los análisis de variables moderadoras generales, evidenciaron que la edad, masa corporal, talla y sexo parecen no influir en los TE. Por otro lado, se evidenció una diferencia significativa entre diseños, el Post Hoc de Tukey mostró que los TE individuales provenientes de diseños experimentales son significativamente más altos $\left(p<0.05^{*}\right)$ que los TE individuales provenientes de diseños cuasiexperimentales y pre-experimentales; es importante recalcar que los tres diseños mostraron un TE global significativo $\left(p<0.05^{*}\right)$ positivo. Dicha diferencia podría explicarse por el hecho de que los TE individuales de diseños experimentales en su mayoría son del tipo de entrenamiento pliometría, este tipo de entrenamiento fue el que mostró el TE global más alto (TE = 0.85); añadido a esto, el TE global del diseño experimental fue el que menos incluyó TE individuales provenientes del tipo de entrenamiento estiramiento, este tipo de entrenamiento fue el que mostró el TE global más bajo (TE = -0.07).

Ahora bien, el análisis de varianza y el Post Hoc de Tukey evidenció que el tipo de entrenamiento estiramiento tiene un TE global $(T E=-0.07)$ significativamente menor $\left(p<0.05^{*}\right)$ que pliometría (TE $\left.=0.85\right)$, vibración $(T E=0.54)$ y "otros" (TE $\left.=0.48\right)$; así mismo, el TE global de pliometría solo es significativamente mayor a estiramiento y "otros". Este hallazgo es uno de los más importantes, pues permite inferir que el tipo de entrenamiento físico que más contribuye a la mejora de la altura de salto de gimnastas es la pliometría. En este sentido, la evidente supremacía de pliometría sobre estiramiento, vibración y "otros", podría explicarse por el principio de especificidad del entrenamiento (McArdle, Katch, y Katch, 2015), pues la pliometría para tren inferior se basa en su mayoría en la ejecución saltos con la máxima potencia posible. Además, la pliometría también posee gran afinidad con la mayoría de los gestos deportivos de gimnasia, pues las ejecuciones consisten en la aceleración y desaceleración rápida de los músculos, creando un ciclo de acortamientoestiramiento; estos ejercicios pueden ayudar a los músculos, el tejido conectivo y el sistema nervioso a pasar eficientemente a través de los ciclos de elongación y contracción que conducen a una mejora de las actuaciones deportivas (Farentinos y Radcliffe, 1999). Estos resultados concuerdan con los meta-análisis de Stojanović, Ristić, McMaster y Milanović, (2017) y de Villarreal, Kellis, Kraemer e Izquierdo (2009), en cuanto que, el entrenamiento de pliometría mejora significativamente la altura de salto de población general y mujeres atletas respectivamente. 
Cuando se analizaron los TE obtenidos en los diferentes estudios que aplicaron entrenamiento pliométrico y diferentes variables moderadoras relacionadas con la prescripción del ejercicio; los resultados de la variable volumen se deben tomar con cautela, pues el volumen de 1710 minutos corresponde al estudio de Mirela, Raducu, Antoanela, Carmen y Laura (2014), este volumen tan alto se debe a que dicho estudio se desarrolló durante un macrociclo de 9 meses de duración, el mismo supera hasta tres veces la cantidad de semanas de las intervención de los demás estudios incluidos. Estos resultados concuerdan con el meta-análisis de Stojanović et al. (2017), quienes evidenciaron que a mayor volumen de entrenamiento mayor TE en mujeres atletas.

En este contexto, la evidencia existente sobre el entrenamiento pliométrico, puede sugerir con cierta base científica, que la inclusión del mismo en el programa de preparación física de los gimnastas, especialmente los de élite, ayudaría a mejorar el rendimiento por medio del mejoramiento de la fuerza explosiva de tren inferior expresada por la altura de salto.

En cuanto al estiramiento, este tipo de entrenamiento mostró un TE global no significativo ( $T E=-0.07, p=0.56$ ), lo que evidencia que no existe una aparente conexión entre la altura de salto de gimnastas y estirar. Estos resultados concuerdan con una revisión sistemática realizada anteriormente (Ulloa-Sánchez, 2020), pues los resultados de dicho estudio muestran que los gimnastas no presentan cambios significativos en la altura de salto después de una intervención de estiramiento; es importante recalcar que al igual que el presente estudio, dicha revisión sistemática incluyó diferentes tipos de estiramiento (EE, ED, FNP). También es fundamental mencionar que, el análisis de variables moderadoras evidenció que aquellos estudios con TE mayores en la altura de salto se relacionan significativamente con menor cantidad de series por sesión. Además, la correlación directa y significativa entre el número de ejercicios por sesión y el rendimiento del salto de gimnastas, se debe tomar con cautela, pues un análisis más profundo de los datos sugiere que dicha correlación directa se debe a los datos del estudio de Montalvo y Dorgo (2019), esto debido a que las dos intervenciones de este estudio se constituyeron entre 15 a 30 ejercicios por sesión, mientras que el rango del resto de intervenciones codificadas fue de 1 a 8 ejercicios por sesión; sabiendo esto, al realizar la correlación de Pearson excluyendo los datos de Montalvo y Dorgo (2019), se obtuvo una correlación inversa, baja y no significativa $(r=-0.26, p=0.15)$. En resumen, aunque estos resultados no son concluyentes, ya que se extraen a partir de un análisis correlacional, podrían sugerir que el estiramiento 
utilizado como una herramienta para mejorar la altura de salto de gimnastas podría no ser efectiva.

Autores como Dallas, Smirniotou, et al. (2014), Di Cagno et al. (2010), Donti, Tsolakis, y Bogdanis (2014), Özengin, Un Yildirim, Baltaci, y Masiulis (2011), Papia et al. (2018) concuerdan en que el tipo de entrenamiento estiramiento a largo plazo, puede provocar adaptaciones específicas como el mantenimiento de la rigidez muscular y, por lo tanto, el rendimiento muscular, esto después de un aumento de la longitud muscular inducido por el estiramiento. En otras palabras, se podría inferir que en los gimnastas, el hecho entrenar la flexibilidad y fuerza explosiva durante los macrociclos de toda su carrera deportiva, disminuya su susceptibilidad posibles efectos adversos del estiramiento sobre la capacidad de salto.

Para el presente meta-análisis fue muy importante incluir este tipo de entrenamiento, pues es parte fundamental del entrenamiento diario de los gimnastas, ya que, al ser un deporte estético, cada ejecución de los elementos en los diferentes aparatos debe tener cierta amplitud de miembros inferiores como lo establece el código de puntuación, lo que significa que gran parte del rendimiento en este deporte depende del rango de movimiento de las articulaciones, especialmente en las que participan los grupos musculares cuádriceps, glúteos, isquiotibiales, iliopsoas y tríceps sural.

Por otro lado, el tipo de entrenamiento vibración, mostró un TE global moderado (TE $=0.54)$ y significativo $\left(p<0.001^{*}\right)$; parece ser un buen tipo de entrenamiento para mejorar la altura de salto de gimnastas, esta hipótesis es respaldada por cinco estudios ejecutados por autores experimentados en intervenciones con muestras constituidas por gimnastas de élite (Dallas, Kirialanis et al., 2014; Dallas, Savvathi et al., 2019; Tsopani et al., 2014; Kinser et al., 2008; Mazo, 2010). Es muy importante mencionar que solo dos estudios tuvieron un diseño de efecto crónico (Dallas, Savvathi et al., 2019; Mazo, 2010), por lo que se recomienda tomar estos resultados con prudencia. El análisis de variables moderadoras evidenció que los minutos por sesión, las series por sesión, la duración de la serie, el número de ejercicios y los Hertz prácticamente no se relacionan con el rendimiento del salto vertical de gimnastas, pues ninguna de estas variables obtuvo valores de $r$ significativos, sin embargo, las sesiones $\left(r=0.80, p<0.001^{*}\right)$ y la media de desplazamiento $(\mathrm{mm})(r=0.71$, $\left.p<0.001^{*}\right)$ tuvieron correlaciones altas y significativas, por lo que permite inferir que entre mayor número de sesiones y media de desplazamiento $(\mathrm{mm})$ mayor altura de salto. Es importante mencionar que los Hertz y la media de desplazamiento son datos que describen la intensidad que fue programada la plataforma de vibración en cada intervención. 
Estos resultados concuerdan con los resultados en población general mostrados en el meta-análisis de Manimmanakorn, Ross, Manimmanakorn, Lucas, y Hamlin (2015), quienes evidenciaron un TE global de 0.68, además, también se concuerda en que a mayor media de desplazamiento $(\mathrm{mm})$ mayor TE. Sin embargo, contrario al presente metaanálisis, dichos autores encontraron que a mayor cantidad de minutos por sesión mayor tamaño de efecto, esta discrepancia podría explicarse por la prevalencia de diseños de efecto crónico de los estudios individuales codificados por Manimmanakorn et al. (2015), es decir, dichos autores codificaron en su mayoría estudios de efecto crónico, mientras que en el presente estudio en su mayoría fueron de efecto agudo.

Autores como Colson, Petit, Hébreard, Tessaro y Pensini (2009) y Annino et al. (2017) explican que el mejoramiento producido por este tipo de entrenamiento se debe a que una intervención de este tipo no produce cambios en los músculos agonistas, sino únicamente en el músculo antagonista. También mencionan que la eficiencia del sistema de articulación también podría estar mediada por el aumento de la temperatura del tejido resultante de la transferibilidad de las ondas ascendentes de vibración.

De acuerdo a estos resultados, se podría sugerir que el entrenamiento en plataforma vibratoria sería un buen complemento en la preparación física de gimnastas; incluso, teniendo en cuenta que tres de los cinco estudios que se codificaron para obtener el TE global tuvieron un diseño de efecto agudo, se podría deducir que su eventual aplicación pre-competencia podría ayudar a mejorar el rendimiento; sin embargo, esta hipótesis habría que tomarla con moderación, pues no se sabe qué tanto podría influir los demás ejercicios del calentamiento gimnástico o, inclusive, el mejor intervalo de tiempo entre la intervención y el momento de competencia. En todo caso, valdría la pena poner a prueba dicha hipótesis por medio de un estudio experimental, por lo que se sugiere a la comunidad científica involucrada en el campo del rendimiento deportivo de gimnasia, considerar este tema en futuros estudios.

Por último, en la categoría "otros" (anexo 6, la cual está conformada por 6 tipos de entrenamiento que no brindaron los suficientes TE individuales para analizarlos estadísticamente por separado (ET, entrenamiento contra resistencia, entrenamiento con cinturones de peso, potenciación post-activación, entrenamiento propioceptivo y battle rope), mostró un TE global moderado y significativo (TE $=0.48, p<0.001^{*}$ ).

Al analizarlos por separado, se puede mencionar que el ET parece provocar un tamaño del efecto fuerte $(T E=1.35)$, dicho entrenamiento se trató de una intervención de 12 semanas de ejercicios de fuerza explosiva de tren inferior en trampolín (Karakollukçu, 
Aslan, Paoli, Bianco y Sahin, 2015). Dicho efecto discrepa lo evidenciado por Jensen, Scott, Krustrup y Mohr (2013), quienes encontraron un efecto adverso $\left(p<0.05^{\star}\right)$ en la altura de salto de gimnastas trampolinistas de élite, después de una simulación de una competencia de esta rama de la gimnasia. Además, tampoco concuerda con un estudio realizado anteriormente, donde se evidenció una disminución significativa de la altura de salto de porristas (pre-test $=26.73 \pm 6.75$, post-test $=24.91 \pm 6.93, p<0.05^{\star}$ ) después de una intervención de entrenamiento en trampolín enfocado en saltos gimnásticos y pliométricos (Ulloa y Hernández, 2020). Sin embargo, es muy probable que la explicación de esta discrepancia se deba a la diferencia de los diseños de los estudios (crónico vs agudo), pues las disminuciones significativas de la altura de salto fueron por diseños de efecto agudo.

En cuanto al entrenamiento contra resistencia, el estudio de Piazza et al. (2014) mostró tres TE individuales grandes (0.88), esta intervención consistió en realizar sentadillas en la máquina Smith a 12 RM, estos hallazgos concuerdan con (Hrysomallis, 2012), dicho autor menciona que en población general el entrenamiento de sentadillas en la máquina Smith aumenta la altura del salto vertical si se aplica de manera apropiada, realizando la intervención enfocado en la potencia muscular. También se concuerda con los estudios de Maio Alves, Rebelo Abrantes y Sampaio (2010) y Mujika, Santisteban y Castagna (2009), ellos reportan incrementos del $5 \%$ al $20 \%$ del salto con contramovimiento después de una intervención de entrenamiento con contra resistencia, quienes explican que dicho entrenamiento produce un aumento de la masa muscular y esto está relacionado con el desarrollo de fuerza explosiva.

En cuanto al entrenamiento con cinturones de peso (weighted belts), los dos TE individuales son moderados (0.51 y 0.76), estos también se obtuvieron del estudio de Piazza et al. (2014), quién implementó ejercicios específicos de potencia de tren inferior con cinturones con un peso del $6 \%$ del peso corporal, estos resultados concuerdan con los estudios de Thompsen, Kackley, Palumbo y Faigenbaum (2007), quienes luego de realizar una intervención de este tipo, encontraron mejoras en la altura de salto en atletas jóvenes femeninas y en un grupo de mujeres con un promedio de edad de 19.7 años respectivamente. Piazza et al. (2014) mencionan que este tipo de entrenamiento puede mejorar la activación y coordinación neuromuscular.

Respecto al método de potenciación post-activación (PAP) (anexo 6), los estudios de Dallas et al. (2018) y Dallas et al. (2019) mostraron un rango de TE individuales entre 0.10 y 0.63 , los ejercicios de potenciación consistieron en la ejecución de saltos específicos de gimnasia y saltos pliométricos (como por ejemplo: 10 rondadas y 2 series de 5 repeticiones 
de saltos agrupados respectivamente). En este tipo de intervenciones se suele medir el efecto en diferentes momentos post-activación, es decir, los autores midieron el efecto inmediato y 3, 6 y 9 minutos después. Al organizar los TE individuales en rangos por cada momento de medición, se evidenció que a los 6 min se mostraron los TE más grandes (TE $=0.26-0.51)$. Se podría conjeturar que estos resultados concuerdan con lo evidenciado en el meta-análisis de Dobbs, Tolusso, Fedewa y Esco (2019), quienes evidenciaron que en sujetos entrenados, el mejor rendimiento en la altura de salto se evidenció entre los 3 y 7 minutos después de un estímulo de PAP.

Finalmente, los dos últimos tipos de entrenamientos que se incluyeron en el presente estudio fueron intervenciones muy particulares (entrenamiento propioceptivo y battle rope), por lo que su exploración apenas está comenzando, esto dificulta discutir sus resultados con literatura existente en la actualidad. En cuanto al entrenamiento propioceptivo, mostró un TE moderado (TE = 0.41), Dobrijević et al. $(\underline{2018})$ menciona que este tipo de entrenamiento está dirigido a la estimulación de los propioceptores (husos musculares, los órganos del tendinosos de Golgi y los corpúsculos de Pacini) con el objetivo de provocar una respuesta motora adecuada, que principalmente contribuye a la estabilidad de las articulaciones y a un rendimiento más preciso y más eficiente de todo tipo de movimientos. Mientras que, la intervención con battle rope mostró un TE grande (TE =1.16), Azab (2019) menciona que este entrenamiento se caracteriza por ser de alta intensidad y que involucra todo el cuerpo, pues se trata de ejecuciones de ejercicios explosivos que consisten en ondular vigorosamente una cuerda especial con la parte superior del cuerpo, este entrenamiento lo han popularizado las personas que realizan CrossFit.

En general, los entrenamientos agrupados en la categoría "otros" mostraron TE individuales muy buenos, sin embargo, estos resultados se deben tomar con reserva, pues hasta el momento, existe un bajo volumen de evidencia científica al respecto. En este sentido, futuros estudios podrían enfocarse en brindar mayor evidencia científica para mostrar los beneficios de estos poco conocidos tipos de entrenamiento físico sobre la altura de salto de gimnastas.

\section{CONCLUSIONES}

1. El tipo de entrenamiento que mostró el mayor TE global fue pliometría ( $\mathrm{N}$ de $\mathrm{TE}=$ 19, TE global $\left.=0.85 ; p<0.001^{*}\right)$, seguido por el entrenamiento en plataforma vibratoria $\left(\mathrm{N}\right.$ de $\mathrm{TE}=19$, TE global $\left.=0.54 ; \mathrm{p}<0.001^{*}\right)$. 
2. Parece que el estiramiento no se relaciona significativamente con la altura de salto de gimnastas ( $\mathrm{N}$ de TE $=47$, TE global $=-0.07 ; \mathrm{p}<0.56$ ).

3. Los tipos de entrenamiento agrupados en la categoría "otros", en su mayoría mostraron TE individuales moderados y grandes (entrenamiento en trampolín: TE = 1.35; entrenamiento contra resistencia: $\mathrm{TE}=0.88$; entrenamiento con cinturones de peso: $\mathrm{TE}=0.51$ y 0.76 ; potenciación post-activación: $\mathrm{TE}=0.10-0.63$; entrenamiento propioceptivo: $\mathrm{TE}=0.41$; battle rope: $\mathrm{TE}=1.16$ ).

\section{APLICACIONES PRÁCTICAS}

Se recomienda implementar un entrenamiento pliométrico de 12 sesiones distribuidas en 4 semanas, 6 series por sesión, 93 saltos por sesión y descansos de 120 segundos entre series. Además, se recomienda implementar este entrenamiento en diferentes ocasiones durante un macrociclo que dure aproximadamente 9 meses.

\section{FORTALEZAS Y LIMITACIONES}

La principal fortaleza del presente estudio de carácter meta-analítico, fue que logró recopilar los principales entrenamientos físicos que mejoran la altura de salto de gimnastas.

Además, contribuyó a establecer bases más concretas sobre la hipótesis de que el estiramiento no afecta significativamente la altura de salto de gimnastas.

Por otro lado, el presente meta-análisis no pudo incluir la totalidad de estudios que evidenciaron el efecto de algún entrenamiento físico sobre la altura de salto, debido a que no presentaron la estadística descriptiva necesaria para su codificación y, aunque se solicitó la información vía correo electrónico, no fue posible lograrlo con todos los estudios.

\section{NO SE DECLARA CONFLICTO DE INTERESES}




\section{REFERENCIAS}

Agostini, B.R., Palomares, E.M.G., Andrade, R.A., Uchôa, F.N.M., y Alves, N. (2017). Analysis of the influence of plyometric training in improving the performance of athletes in rhythmic gymnastics. Motricidade, 13(2), 71-80. doi: https://doi.org/10.6063/motricidade.9770

Annino, G., lellamo, F., Palazzo, F., Fusco, A., Lombardo, M., Campoli, F., y Padua, E. (2017). Acute changes in neuromuscular activity in vertical jump and flexibility after exposure to whole body vibration. Medicine, 96(33), e7629. doi: https://doi.org/10.1097/MD.0000000000007629

Azab, M. (2019). Effects of Battle Rope Exercises on Power and Leaping Ability in Rhythmic Gymnasticsfor Female College Students. University Annals, Series Physical Education y Sport/Science, Movement \& Health, 19(suppl. 2), 266-271. Recuperado de https://www.analefefs.ro/en/anale-fefs/2019/i2suplement/peautori/MANAL AZAB.PDF

Bogdanis, G. C., Donti, O., Papia, A., Donti, A., Apostolidis, N., y Sands, W. A. (2019). Effect of Plyometric Training on Jumping, Sprinting and Change of Direction Speed in Child Female Athletes. Sports, 7(5), e116. doi: https://doi.org/10.3390/sports7050116

Bogdanis, G.C., Donti, O., Tsolakis, C., Smilios, I., y Bishop, D.J. (2019). Intermittent But Not Continuous Static Stretching Improves Subsequent Vertical Jump Performance In Flexibility-Trained Athletes. Journal of Strength and Conditioning Research, 33(1), 203-210. doi: https://doi.org/10.1519/JSC.0000000000001870

Bradshaw, E. J., y Le Rossignol, P. (2004). Anthropometric and biomechanical field measures of floor and vault ability in 8 to 14 year old talent-selected gymnasts. Sports Biomechanics, 3(2), 249-262. https://doi.org/10.1080/14763140408522844

Campbell, D., y Stanley, J. (1970). Diseños experimentales y cuasiexperimentales en la investigación social ( $2^{\mathrm{a}}$ ed.). Madrid, España: AMORRORTU.

Chu, D.A., y Myer, G.D. (2016). Pliometría: Ejercicios pliométricos para un entrenamiento completo. Barcelona, España: Editorial Paidotribo.

Colson, S.S., Petit, P.-D., Hébreard, L., Tessaro, J., y Pensini, M. (2009). Whole body vibration does not enhance muscle activation. International Journal of Sports Medicine, 30(12), 841-844. doi: https://doi.org/10.1055/s-0029-1234082

Dallas, G., Kirialanis, P., y Mellos, V. (2014). The Acute Effect of Whole Body Vibration Training Oh Flexibility and Explosive Strength of Young Gymnasts. Biology of Sport: 
a quarterly journal of sport and exercise sciences, 31(3), 233-237. doi: https://doi.org/10.5604/20831862.1111852

Dallas, G., Mavvidis, A., Kosmadaki, I., Tsoumani, S., y Dallas, K. (2019). The Post Activation Potentiation Effect of Two Different Conditioning Stimuli on Drop Jump Parameters on Young Female Artistic Gymnasts. Science of Gymnastics Journal, 11(1), 103-114. Recuperado de https://www.fsp.uni-li.si/mma/-/20190224190857/

Dallas, G., Pappas, P., Ntallas, C., y Paradisis, G. (2018). The post-activation effect with two different conditioning stimuli on drop jump performance in pre-adolescent female gymnasts. Journal of Physical Education \& Sport, 18(4), 2368-2374. doi: https://doi.org/10.7752/jpes.2018.04357

Dallas, G., Savvathi, A., Dallas, K., y Maridaki, M. (2019). The Effect of 6-Weeks Whole Body Vibration on Muscular Performance on Young Noncompetitive Female Artistic Gymnasts. Science of Gymnastics Journal, 11(2), 151-162. Recuperado de https://www.fsp.uni-lj.si/mma//20190602135709/\#: :text=The\%20findings\%20of\%20this\%20study,traditional\%20st rength $\% 20$ training $\% 20$ (NVG).

Dallas, G., Smirniotou, A., Tsiganos, G., Tsopani, D., Di, C., y Tsolakis, Ch. (2014). Acute effect of different stretching methods on flexibility and jumping performance in competitive artistic gymnasts. The Journal of sports medicine and physical fitness, 54(6), 683-690. Recuperado de https://pubmed.ncbi.nlm.nih.gov/25350026/

Di Cagno, A., Baldari, C., Battaglia, C., Gallotta, M. C., Videira, M., Piazza, M., y Guidetti, L. (2010). Preexercise Static Stretching Effect on Leaping Performance in Elite Rhythmic Gymnasts. Journal of Strength \& Conditioning Research, 24(8), 1995-2000. doi: https://doi.org/10.1519/JSC.0b013e3181e34811

Dobbs, W.C., Tolusso, D.V., Fedewa, M.V., y Esco, M.R. (2019). Effect of Postactivation Potentiation on Explosive Vertical Jump: A Systematic Review and Meta-Analysis. Journal of Strength and Conditioning Research, 33(7), 2009-2018. doi: https://doi.org/10.1519/JSC.0000000000002750

Dobrijević, S.M., Moskovljević, L., Marković, M., y Dabović, M. (2018). Effects of Proprioceptive Training on Explosive Strenght, Aglility and Coordination of Young Rhythmic Gymnasts. Physical Culture, 72(2), 71-79. doi: https://doi.org/10.5937/zkul1801071D

Donti, O., Tsolakis, C., y Bogdanis, G. C. (2014). Effects of Baseline Levels of Flexibility and Vertical Jump Ability on Performance Following Different Volumes of Static Stretching 
and Potentiating Exercises in Elite Gymnasts. Journal of Sports Science \& Medicine, 13(1), 105-113. Recuperado de https://pubmed.ncbi.nlm.nih.gov/24570613/

Farentinos, R., y Radcliffe, J. (1999). High-Powered Plyometrics. Champaign, Estados Unidos: Human Kinetics.

Ferrara, F., Forte, D., Senatore, B., y D'elia, F. (2019). The relationship between stretching and jumping in artistic gymnastics. Journal of Physical Education \& Sport, 19(Suppl. 5), 1856-1858. doi: https://doi.org/10.7752/jpes.2019.s5274

Ferri-Caruana, A., Roig-Ballester, N., y Romagnoli, M. (2020). Effect of Dynamic Range of Motion and Static Stretching Techniques on Flexibility, Strength and Jump Performance in Female Gymnasts. Science of Gymnastics Journal, 12(1), 87-100. Recuperado de https://search.proquest.com/openview/0f9aae435014231c1a59cae2acc977ae/1?pqorigsite $=$ gscholar $\& \mathrm{cbl}=666318$

Hall, E., Bishop, D. C., y Gee, T. I. (2016). Effect of Plyometric Training on Handspring Vault Performance and Functional Power in Youth Female Gymnasts. PLoS ONE, 11(2), e0148790. doi: https://doi.org/10.1371/journal.pone.0148790

Harper, E.N. (2011). The Effects of Static and Dynamic Stretching on Competitive Gymnasts' Split Jump Performance (Tesis de maestría). Miami University, Estados Unidos.

Recuperado

de https://etd.ohiolink.edu/apexprod/rws etd/send file/send?accession=miami1312391 877\&disposition=inline

Hrysomallis, C. (2012). The effectiveness of resisted movement training on sprinting and jumping performance. Journal of Strength and Conditioning Research, 26(1), 299-306. doi: $\underline{\text { https://doi.org/10.1519/JSC.0b013e3182185186 }}$

Jensen, P., Scott, S., Krustrup, P., y Mohr, M. (2013). Physiological responses and performance in a simulated trampoline gymnastics competition in elite male gymnasts. Journal of Sports Sciences, 31(16), 1761-1769. doi: https://doi.org/10.1080/02640414.2013.803591

Johnson, A.W., Warcup, C.N., Seeley, M.K., Eggett, D., y Feland, J.B. (2019). The acute effects of stretching with vibration on dynamic flexibility in young female gymnasts. The Journal of Sports Medicine and Physical Fitness, 59(2), 210-216. doi: https://doi.org/10.23736/S0022-4707.18.08290-7

Karakollukçu, M., Aslan, C.S., Paoli, A., Bianco, A., y Sahin, F.N. (2015). Effects of mini trampoline exercise on male gymnasts' physiological parameters: A pilot study. The 
Journal of Sports Medicine and Physical Fitness, 55(7-8), 730-734. Recuperado de https://pubmed.ncbi.nlm.nih.gov/24921617/

Kinser, A.M., Ramsey, M.W., O'Bryant, H.S., Ayres, C.A., Sands, W.A., y Stone, M.H. (2008). Vibration and Stretching Effects on Flexibility and Explosive Strength in Young Gymnasts. Medicine \& Science in Sports \& Exercise, 40(1), 133-140. doi: https://doi.org/10.1249/mss.0b013e3181586b13

Leyton, M., Campo, V.L., Sabido, R., y Morenas, J. (2015). Perfil y diferencias antropométricas y físicas de gimnastas de tecnificación de las modalidades de artística y rítmica. Retos: nuevas tendencias en Educación Física, Deportes y Recreación, 21, 58-62. doi: https://doi.org/10.47197/retos.v0i21.34606

Liberati, A., Altman, D.G., Tetzlaff, J., Mulrow, C., Gøtzsche, P.C., loannidis, J.P.A., Clarke, M. ... y Moher, D. (2009). The PRISMA Statement for Reporting Systematic Reviews and Meta-Analyses of Studies That Evaluate Health Care Interventions: Explanation and Elaboration. PLoS Medicine, 6(7), e1000100. doi: https://doi.org/10.1371/journal.pmed.1000100

Maio Alves, J.M.V., Rebelo, A.N., Abrantes, C., y Sampaio, J. (2010). Short-term effects of complex and contrast training in soccer players' vertical jump, sprint, and agility abilities. Journal of Strength and Conditioning Research, 24(4), 936-941. doi: https://doi.org/10.1519/JSC.0b013e3181c7c5fd

Manimmanakorn, N., Ross, J.J., Manimmanakorn, A., Lucas, S.J.E., y Hamlin, M. J. (2015). Effect of Whole-Body Vibration Therapy on Performance Recovery. International Journal of Sports Physiology \& Performance, 10(3), 388-395. doi: https://doi.org/10.1123/ijspp.2014-0225

Mazo, L. (2010). Efectos del entrenamiento con plataforma vibratoria en gimnastas de alto rendimiento. Kronos: revista universitaria de la actividad física y el deporte, 9(18), 6975.

Recuperado

de https://abacus.universidadeuropea.es/bitstream/handle/11268/3151/Kronos 18 8.pd f?sequence $=1$ \&isAllowed $=y$

McArdle, W.D., Katch, F.I., y Katch, V.L. (2015). Fisiología del ejercicio: Nutrición, rendimiento y salud (8 ${ }^{\mathrm{a}}$ ed.). España: Wolters Kluwer Health.

Mirela, D., Raducu, P., Antoanela, O., Carmen, T., y Laura, G. (2014). Plyometric Exercises to Improve Explosive Power in Artistic Gymnastics. Ovidius University Annals, Series Physical Education \& Sport/Science, Movement \& Health, 14(2) 381-386. Recuperado de https://www.analefefs.ro/anale-fefs/2014/i2 supp/pe-autori/7.pdf 
Mlsnová, G., y Luptáková, J. (2017). Influence of Plyometrics on Jump Capabilities in Technical and Aesthetical Sports. Acta Facultatis Educationis Physicae Universitatis Comenianae, 57(1), 76-88. Recuperado de https://sciendo.com/issue/afepuc/57/1

Montalvo, S., y Dorgo, S. (2019). The effect of different stretching protocols on vertical jump measures in college age gymnasts. Journal of Sports Medicine and Physical Fitness, 59(12), 1956-1962. doi: https://doi.org/10.23736/S0022-4707.19.09561-6

Mujika, I., Santisteban, J., y Castagna, C. (2009). In-season effect of short-term sprint and power training programs on elite junior soccer players. Journal of Strength and Conditioning Research, 23(9), 2581-2587. doi: https://doi.org/10.1519/JSC.0b013e3181bc1aac

Özengin, N., Un Yildirim, N., Baltaci, G., y Masiulis, N. (2011). Acute Effects of Different Stretching Durations on Vertical Jump Performance in Rhythmic Gymnasts. Baltic Journal of Sport and Health Sciences, 3(82), 16-22. Recuperado de https://journals.Isu.It/baltic-journal-of-sport-health/article/view/316/312

Papia, K., Bogdanis, G. C., Toubekis, A., Donti, A., y Donti, O. (2018). Acute Effects of Prolonged Static Stretching on Jumping Performance and Range of Motion in Young Female Gymnasts. Science of Gymnastics Journal, 10(2), 217-226. Recuperado de https://bit.ly/2NvhgQt

Pérez, P., y Llana, S. (2015). Biomecánica básica aplicada a la actividad física y el deporte. Barcelona, España: Paidotribo. Recuperado de https://dialnet.unirioja.es/servlet/libro?codigo=564214

Piazza, M., Battaglia, C., Fiorilli, G., Innocenti, G., Iuliano, E., Aquino, G., Calcagno, G., ... Di Cagno, A. (2014). Effects of resistance training on jumping performance in preadolescent rhythmic gymnasts: A randomized controlled study. Italian Journal Of Anatomy And Embryology, 119(1), 10-19. Recuperado de https://core.ac.uk/download/pdf/301568914.pdf

Rodríguez, I., Molinuelo, J.S., Rivilla-García, J., Ródenas, A.B., y Galán, M.H. (2010). Evolución y relación de la capacidad de salto y amortiguación en gimnastas de rítmica de alto nivel. Cuadernos de Psicología del Deporte, 10(2). Recuperado de https://revistas.um.es/cpd/article/view/112601

Romero, E., Aymara, V.D. y Rojas, J.M. (2020). Effects of plyometrics on the explosive strength of lower limbs in senior freestyle wrestling. Revista Cubana de Investigaciones Biomédicas, 39(1), e364. Recuperado de https://www.medigraphic.com/pdfs/revcubinvbio/cib-2020/cib201r.pdf 
Silva, G.F., Almeida, A.R., Rodrigues, S.A., Szmuchrowski, L.A., da Silva, R.A.D., y Drummond, M.D.M. (2018). The Acute Effect of a Sport-Specific Stretching Routine on the Performance of Vertical Jumps in Rhythmic Gymnasts. Journal of Exercise Physiology Online, 21(2), 30-39. Recuperado de https://www.asep.org/asep/asep/JEPonlineAPRIL2018 Drummond.pdf

Stojanović, E., Ristić, V., McMaster, D.T., y Milanović, Z. (2017). Effect of Plyometric Training on Vertical Jump Performance in Female Athletes: A Systematic Review and Meta-Analysis. Sports Medicine, 47(5), 975-986. doi: https://doi.org/10.1007/s40279016-0634-6

Taktak, F., TakTak, I., y Shephard, R.J. (2013). A controlled trial of plyometric training for rhythmic female gymnasts. The Health \& Fitness Journal of Canada, 6(3), 123-131. doi: https://doi.org/10.14288/hfjc.v6i3.142

Thomas, J.R., y French, K.E. (1986). The use of meta-analysis in exercise and sport: A tutorial. Research Quarterly for Exercise and Sport, 57(3), 196-204. doi: https://doi.org/10.1080/02701367.1986.10605397

Thompsen, A.G., Kackley, T., Palumbo, M.A., y Faigenbaum, A.D. (2007). Acute effects of different warm-up protocols with and without a weighted vest on jumping performance in athletic women. Journal of strength and conditioning research, 21(1), 52-56. doi: https://doi.org/10.1519/00124278-200702000-00010

Tsopani, D., Dallas, G, Tsiganos, G., Papouliakos, S., Di Cagno, A., Korres, G., Rigo, M y Korres, S. (2014). Short-term effect of whole-body vibration training on balance, flexibility and lower limb explosive strength in elite rhythmic gymnasts. Human Movement Science, 33, 149-158. doi: https://doi.org/10.1016/j.humov.2013.07.023

Ulloa-Sánchez, P. (2020). El efecto agudo de diferentes tipos de estiramiento sobre la altura de salto de gimnastas: Revisión sistemática. MHSalud: Revista en Ciencias del Movimiento Humano y Salud, 17(2), 1-20. doi: https://doi.org/10.15359/mhs.17-2.7

Ulloa, P., y Hernández, J. (2020). El efecto agudo del entrenamiento en trampolín sobre la estabilidad, la altura de salto y la velocidad de carrera en 20 metros de porristas. Pensar en Movimiento: Revista de Ciencias del Ejercicio y la Salud, 18(1), e38560. doi: https://doi.org/10.15517/pensarmov.v18i1.38560

de Villarreal, E.S.-S., Kellis, E., Kraemer, W.J., e Izquierdo, M. (2009). Determining Variables of Plyometric Training for Improving Vertical Jump Height Performance: A Meta-Analysis. The Journal of Strength \& Conditioning Research, 23(2), 495-506. doi: https://doi.org/10.1519/JSC.0b013e318196b7c6 


\section{ANEXOS}

Anexo 1.

Tabla de descripción de los estudios codificados para el tipo de entrenamiento pliometría.

\begin{tabular}{|c|c|c|c|c|c|c|}
\hline Estudio & Edad $\bar{X}$ & Diseño & $\mathbf{N}$ & Semanas & $\begin{array}{l}\text { Días por } \\
\text { semana }\end{array}$ & Saltos evaluados \\
\hline Hall et al. (2016) & 12.5 & Experimental & 20 & 6 & 2 & SJ \\
\hline Mlsnová y Luptáková (2017) & 12.4 & Cuasiexperimental & 30 & 30 & 2 & CMJ Y CMJS \\
\hline Taktak et al. (2013) & 18.2 & Experimental & 20 & 4 & 3 & SJ, CMJ, CMJA y DJ \\
\hline $\begin{array}{l}\text { Bogdanis, Donti, Papia et al. } \\
(\underline{2019})\end{array}$ & 8.1 & Experimental & 33 & 8 & 2 & CMJ y DJ \\
\hline Agostini et al. (2017) & 15.3 & Experimental & 30 & 52 & 2 & CMJ \\
\hline
\end{tabular}

Nota: $\mathrm{N}$ = número de sujetos; $\mathrm{SJ}$ = squat jump, $\mathrm{CMJ}$ = salto con contramovimiento; $\mathrm{DJ}=$ drop jump. Fuente: elaboración propia. 
Anexo 2.

Tabla de descripción de los estudios codificados para el tipo de entrenamiento estiramiento.

\begin{tabular}{|c|c|c|c|c|c|c|c|c|}
\hline Estudio & Edad $\bar{X}$ & Diseño & $\mathbf{N}$ & $\begin{array}{c}\text { Tipo de } \\
\text { estiramiento }\end{array}$ & $\begin{array}{c}\text { Duración de } \\
\text { cada } \\
\text { estiramiento } \\
\bar{X}(s) \\
\end{array}$ & Músculos estirados & $\begin{array}{c}\mathrm{N} \text { de } \\
\text { ejercicios }\end{array}$ & $\begin{array}{c}\text { Saltos } \\
\text { evaluados }\end{array}$ \\
\hline Harper (2011) & 11.8 & Pre-experimental & 12 & ED & - & - & 5 & Salto split \\
\hline Harper (2011) & 11.8 & Pre-experimental & 12 & EE & 48 & $\begin{array}{c}\text { Cuádriceps, flexores } \\
\text { plantares, } \\
\text { isquiotibiales y } \\
\text { flexores de la cadera }\end{array}$ & 4 & Salto split \\
\hline $\begin{array}{l}\text { Özengin et al. } \\
(\underline{2011})\end{array}$ & 10 & Pre-experimental & 27 & EE & 15 & $\begin{array}{c}\text { Flexores de la } \\
\text { cadera, isquiotibiales } \\
\text { y gastrocnemio }\end{array}$ & 3 & SJ \\
\hline $\begin{array}{l}\text { Özengin et al. } \\
(\underline{2011})\end{array}$ & 10 & Pre-experimental & 27 & $\mathrm{EE}$ & 30 & $\begin{array}{c}\text { Flexores de la } \\
\text { cadera, isquiotibiales } \\
\text { y gastrocnemio }\end{array}$ & 3 & SJ \\
\hline $\begin{array}{l}\text { Di Cagno et al. } \\
\text { (2010) }\end{array}$ & 14.16 & Pre-experimental & 38 & EE & 30 & $\begin{array}{l}\text { Isquiotibial, } \\
\text { gastrocnemio y } \\
\text { cuádriceps }\end{array}$ & 4 & $\begin{array}{l}\text { SJ, CMJ, } \\
\text { Multisaltos, } \\
\text { Saltos split }\end{array}$ \\
\hline $\begin{array}{l}\text { G. Dallas, } \\
\text { Smirniotou, et al., } \\
\text { 2014) }\end{array}$ & 21.83 & Pre-experimental & 18 & $\mathrm{EE}$ & 15 & $\begin{array}{l}\text { Isquiotibiales, } \\
\text { cuádriceps, sóleo y } \\
\text { gastrocnemio }\end{array}$ & 3 & SJ y CMJ \\
\hline $\begin{array}{l}\text { G. Dallas, } \\
\text { Smirniotou, et al., } \\
\text { 2014) }\end{array}$ & 21.83 & Pre-experimental & 18 & $\begin{array}{c}\text { EE + } \\
\text { vibración }\end{array}$ & 15 & $\begin{array}{l}\text { Isquiotibiales, } \\
\text { cuádriceps, sóleo y } \\
\text { gastrocnemio }\end{array}$ & 3 & SJ y CMJ \\
\hline $\begin{array}{l}\text { G. Dallas, } \\
\text { Smirniotou, et al., } \\
\text { 2014) }\end{array}$ & 21.83 & Pre-experimental & 18 & FNP & 15 & $\begin{array}{l}\text { Isquiotibiales, } \\
\text { cuádriceps, sóleo y } \\
\text { gastrocnemio }\end{array}$ & 3 & SJ y CMJ \\
\hline
\end{tabular}

Nota: $\mathrm{N}$ = número de sujetos; $\mathrm{EE}$ = estiramiento estático; ED = estiramiento dinámico; FNP = estiramiento de facilitación neuromuscular propioceptiva; SJ = squat jump, $\mathrm{CMJ}=$ salto con contramovimiento; DJ = drop jump. Fuente: elaboración propia. 
Anexo 3.

Continuación del anexo 1.

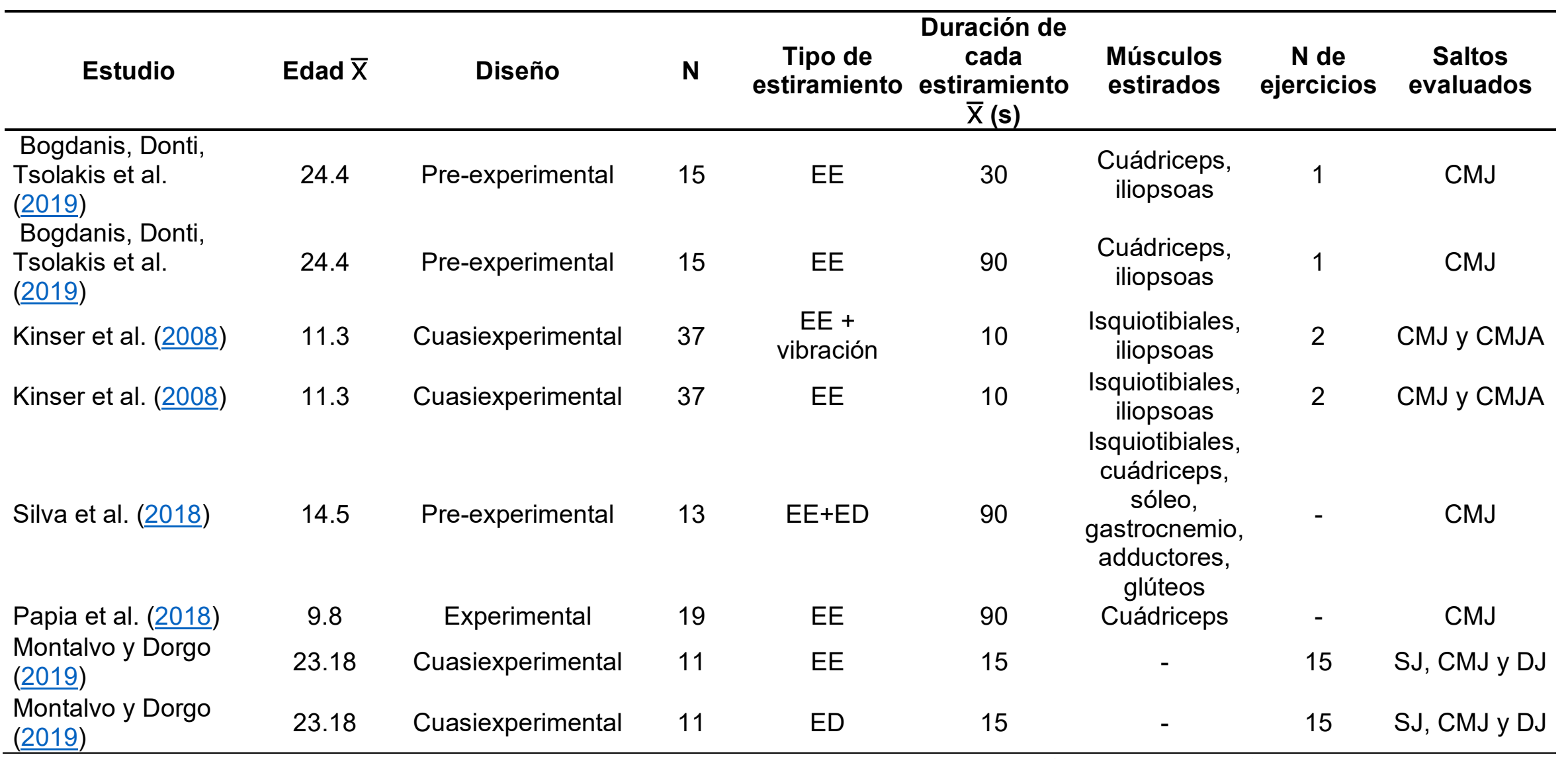

Nota: $\mathrm{N}$ = número de sujetos; $\mathrm{EE}$ = estiramiento estático; $\mathrm{ED}=$ estiramiento dinámico; $\mathrm{SJ}$ = squat jump, $\mathrm{CMJ}=\mathrm{salto}$ con contramovimiento; CMJA = salto con contramovimiento con swing arms; DJ = drop jump. Fuente: elaboración propia. 
Anexo 4.

Continuación del anexo 1.

\begin{tabular}{|c|c|c|c|c|c|c|c|c|}
\hline Estudio & Edad $\bar{X}$ & Diseño & $\mathbf{N}$ & $\begin{array}{c}\text { Tipo de } \\
\text { estiramiento }\end{array}$ & $\begin{array}{c}\text { Duración de } \\
\text { cada } \\
\text { estiramiento } \\
\bar{X}(s) \\
\end{array}$ & Músculos estirados & $\begin{array}{c}\mathrm{N} \text { de } \\
\text { ejercicios }\end{array}$ & $\begin{array}{c}\text { Saltos } \\
\text { evaluados }\end{array}$ \\
\hline $\begin{array}{l}\text { Montalvo y } \\
\text { Dorgo (2019) }\end{array}$ & 23.18 & Cuasiexperimental & 11 & $\mathrm{EE}+\mathrm{ED}$ & 30 & - & 30 & SJ, CMJ y DJ \\
\hline $\begin{array}{l}\text { Ferri-Caruana } \\
\text { et al. (2020) }\end{array}$ & 13 & Pre-experimental & 9 & EE & 90 & $\begin{array}{l}\text { Isquiotibiales, } \\
\text { cuádriceps, sóleo, } \\
\text { gastrocnemio, } \\
\text { adductores, glúteo }\end{array}$ & 4 & SJ \\
\hline $\begin{array}{l}\text { Ferri-Caruana } \\
\text { et al. (2020) }\end{array}$ & 13 & Pre-experimental & 9 & ED & 15 & $\begin{array}{l}\text { Isquiotibiales, } \\
\text { cuádriceps, sóleo, } \\
\text { gastrocnemio, } \\
\text { adductores, glúteos }\end{array}$ & 8 & SJ \\
\hline $\begin{array}{l}\text { Ferrara et al. } \\
(\underline{2019})\end{array}$ & 9.4 & Experimental & 5 & EE & 15 & $\begin{array}{l}\text { Gastrocnemio y } \\
\text { cuádriceps }\end{array}$ & - & CMJA \\
\hline $\begin{array}{l}\text { Ferrara et al. } \\
(\underline{2019)}\end{array}$ & 10.4 & Experimental & 5 & ED & 15 & $\begin{array}{l}\text { Gastrocnemio y } \\
\text { cuádriceps }\end{array}$ & - & CMJA \\
\hline $\begin{array}{l}\text { Johnson et al. } \\
(\underline{2019)})\end{array}$ & 11.5 & Experimental & 14 & $\begin{array}{c}\text { EE + } \\
\text { vibración }\end{array}$ & 30 & $\begin{array}{l}\text { Flexores de la cadera, } \\
\text { isquiotibiales, glúteo, } \\
\text { cuádriceps }\end{array}$ & 3 & Salto vertical \\
\hline $\begin{array}{l}\text { Johnson et al. } \\
(\underline{2019)}\end{array}$ & 11.5 & Experimental & 13 & EE & 30 & $\begin{array}{l}\text { Flexores de la cadera, } \\
\text { isquiotibiales, glúteo, } \\
\text { cuádriceps }\end{array}$ & 3 & Salto Vertical \\
\hline
\end{tabular}

Nota: $\mathrm{N}$ = número de sujetos; $\mathrm{EE}$ = estiramiento estático; $\mathrm{ED}=$ estiramiento dinámico; $\mathrm{SJ}$ = squat jump, $\mathrm{CMJ}=$ salto con contramovimiento; $\mathrm{CMJA}=$ salto con contramovimiento con swing arms; DJ = drop jump. Fuente: elaboración propia. 
Anexo 5.

Tabla de descripción de los estudios codificados para el tipo de entrenamiento vibración

\begin{tabular}{|c|c|c|c|c|c|c|c|c|}
\hline Estudio & Edad $\bar{X}$ & Diseño & $\mathbf{N}$ & $\begin{array}{c}\text { Duración } \\
\text { de la serie } \\
\text { (s) }\end{array}$ & $\begin{array}{l}\text { Número } \\
\text { de } \\
\text { ejercicios }\end{array}$ & Hertz & $\begin{array}{c}\text { Media de } \\
\text { desplazamiento } \\
(\mathrm{mm})\end{array}$ & $\begin{array}{c}\text { Saltos } \\
\text { evaluados }\end{array}$ \\
\hline Kinser et al. (2008) & 10.6 & Cuasiexperimental & 37 & 10 & 2 & 30 & 2 & CMJA \\
\hline Dallas et al. (2014) & 9.22 & Experimental & 34 & 120 & 3 & 30 & 2 & SJ y CMJ \\
\hline Tsopani et al. (2014) & 17.54 & Pre-experimental & 11 & 75 & 5 & 30 & 2 & SJ y CMJ \\
\hline Mazo $(\underline{2010})$ & 21.5 & Pre-experimental & 7 & 60 & 1 & 45 & 1.8 & $\begin{array}{l}\text { SJ, CMJ y } \\
\text { Multisaltos }\end{array}$ \\
\hline Dallas et al. (2019) & 9.7 & Experimental & 12 & 30 & 3 & 30 & 2.5 & SJ y CMJ \\
\hline
\end{tabular}

Nota: $\mathrm{N}$ = número de sujetos; $\mathrm{SJ}=$ squat jump, $\mathrm{CMJ}$ = salto con contramovimiento; DJ = drop jump. Fuente: elaboración propia. 
Anexo 6.

Tabla de descripción de los estudios codificados y agrupados en la categoría "otros"

\begin{tabular}{|c|c|c|c|c|c|c|}
\hline Estudio & Edad $\bar{X}$ & Diseño & $\mathbf{N}$ & Semanas & Entrenamiento & Saltos evaluados \\
\hline $\begin{array}{l}\text { Karakollukçu et al. } \\
(\underline{2015})\end{array}$ & 22.17 & Cuasiexperimenta & 20 & 12 & Tumbling & Salto vertical \\
\hline Piazza et al. (2014) & 11.9 & Pre-experimental & 18 & 6 & $\begin{array}{c}\text { Entrenamiento de } \\
\text { contra resistencia: } \\
\text { Sentadillas con pesas } \\
\text { a } 12 \mathrm{RM}\end{array}$ & SJ y CMJ \\
\hline Piazza et al. (2014) & 12 & Pre-experimental & 19 & 6 & $\begin{array}{c}\text { Weighted Belts } \\
\text { (cinturones con peso } \\
\text { del } 6 \% \text { de la masa } \\
\text { corporal) }\end{array}$ & SJ y CMJ \\
\hline Dallas et al. (2018) & 11.75 & Pre-experimental & 29 & - & $\begin{array}{l}\text { Potenciación post- } \\
\text { activación }\end{array}$ & DJ \\
\hline Dallas et al. (2019) & $11.75 F$ & Pre-experimental & 19 & - & $\begin{array}{l}\text { Potenciación post- } \\
\text { activación }\end{array}$ & DJ \\
\hline Azab (2019) & 19.47 & Experimental & 15 & 10 & Battle Rope & CMJA \\
\hline
\end{tabular}

Nota: $\mathrm{F}=$ mujeres; $\mathrm{M}$ = hombres; $\mathrm{N}$ = número de sujetos; $\mathrm{SJ}=$ squat jump, $\mathrm{CMJ}=$ salto con contramovimiento; $\mathrm{CMJA}=$ salto con contramovimiento con swing arms. Fuente: elaboración propia. 